[0212-7199 (2008) 25: 6; pp 269-274] ANALES DE MEDICINA INTERNA Copyright (C) 2008 ARAN EDICIONES, S.L.

AN. MED. INTERNA (Madrid) Vol. 25, N. ${ }^{\circ}$ 6, pp. 269-274, 2008

\section{Estudio del consumo de fármacos inadecuados o no indicados en el anciano que ingresa en un Servicio de Medicina Interna}

\author{
F. BLASCO PATIÑO, R. PÉREZ MAESTU ${ }^{1}$, J. MARTÍNEZ LÓPEZ DE LETONA', \\ A. I. JIMÉNEZ' ${ }^{1}$ M. J. GARCÍA NAVARRO ${ }^{1}$
}

Servicio de Medicina Interna. Hospital de Torrevieja. Alicante. ${ }^{1}$ Hospital Puerta de Hierro. Madrid
STUDY OF THE CONSUMPTION OF INNAPROPIATE MEDICAMENTS IN ELDER HOSPITALIZED IN THE INTERNAL MEDICINE SERVICE

\section{RESUMEN}

Introducción: Los pacientes ancianos se caracterizan por el alto grado de polimedicación. Este factor se ha mostrado el más importante en la aparición de efectos adversos (EAF). El estudio de los fármacos ayuda a establecer cuales son inapropiados y por lo tanto se deben retirar, disminuyendo de esta forma la posibilidad de EAF.

Material y Métodos: Estudio prospectivo y observacional. Criterio de inclusión: pacientes de > 64 años que ingresan en Medicina Interna. Se definió como fármaco inapropiado los medicamentos de baja utilidad terapéutica (UTB), los no indicados, y los inadecuados para el anciano. Mediante el programa informático SPSS 11.5 se analizaron las posibles variables relacionadas con el consumo

Resultados: Se incluyeron en el estudio 172 ancianos. La media de medicamentos por persona y día fue de 5,34 (0-15). El 52,5\% consumían un fármaco inapropiado (36,6\% inadecuado para el anciano, $15 \%$ no indicado y $12 \%$ UTBs). El análisis multivariante asocia el consumo de estos fármacos a un mayor número de patologías $(\mathrm{p}<0,012)$, a un mayor consumo de medicamentos $(\mathrm{p}<0,001)$ y a la procedencia de residencias de ancianos $(\mathrm{p}<0,001)$. Sólo el consumo de fármacos no adecuados para el anciano se asocia a aumento de EAF

Conclusión: La mitad de los ancianos toma al menos un fármaco de forma innecesaria, y la mayoría de estos fármacos favorece la aparición de EAF.

PALABRAS CLAVE: Efectos adversos. Medicamentos inapropiados. Anciano. Polimedicación.

\section{ABSTRACT}

Background: The elderly patients are characterized by the high degree of polymedication. This factor is the most important in the appearance of adverse effects (EAD). The study of the medicaments helps to establish which are inappropriate and therefore they must move back, diminishing of this form EAD's possibility. on of incorporation: patients of $>64$ years old, hospitalized in an Internal Medicine Service. There were defined as inappropriate medicament the medicines of low therapeutic utility, them not indicated, and the inadequate ones for the elder. By means of the SPSS 11.5 program the possible variables related with the consumption were analyzed.

Results: 172 elders were included in the study. The average of medicines for person and day belonged to $5.34(0-15) .52 .5 \%$ was consuming an inappropriate medicament (36.6\% inadequate for the elder, $15 \%$ not indicated, and $12 \%$ UTBS). The analysis multivariant associates the consumption of these medicaments with a major number of diseases $(p<$ $0.012)$, to a major consumption of medicines $(p<0.001)$ and to the origin of the nursing residences $(p<0.001)$. Only the consumption of medicaments not adapted for the elder is associated with increase of EAD.

Conclusion: The half of the elders takes at least a medicament of unnecessary form, and the majority of these medicaments favors appearance of $E A D$.

KEY WORDS: Adverse drugs events. Innapropiate medicaments. Elder. Polymedication.
Patients and Method: Prospective and observacional Study. Criteri-

Blasco Patiño F, Pérez Maestu R, Martínez López de Letona J, Jiménez AI, García Navarro MJ, Cornide L. Estudio del consumo de fármacos inadecuados o no indicados en el anciano que ingresa en un Servicio de Medicina Interna. An Med Interna (Madrid) 2008; $25: 269-274$.

\section{INTRODUCCIÓN}

El consumo global de fármacos en los ancianos en los países industrializados se caracteriza por dos hechos básicos; de un lado el marcado incremento, en cuanto a cantidad, evidenciado a lo largo de las ultimas décadas, situándose la media en 4,2-8 fármacos/persona/día (1-3), y de otro lado el tipo de fármacos, no todos están indicados para sus patologías, estimándose que el $97 \%$ de los ancianos que viven en residencia y el $61 \%$ de los que viven en domicilio (4) consumen un fármaco inapropiado o inadecuado.
Teniendo en cuenta que el número total de fármacos que consume una persona es el principal factor asociado a la aparición de Efectos Adversos por Fármacos (EAF), estimar cuantos son innecesarios e inadecuados y los factores que se asocian al consumo de los mismos es el primer paso para conseguir una adecuada prescripción, y para disminuir los EAF que en la actualidad suponen entre un 7,2-14\% $(5,6)$ de ingresos de ancianos en los Servicios de Medicina Interna en España.

El objetivo del presente estudio es establecer el porcentaje de ancianos que consumen este tipo de fármacos entre los que ingresan en un Servicio de Medicina Interna y la importancia

Trabajo aceptado: 14 de febrero de 2008 
que este consumo pueda tener en relación con el incremento de efectos adversos.

\section{MATERIAL Y MÉTODOS}

Estudio prospectivo llevado a cabo sobre pacientes que ingresaron por cualquier motivo en el Servicio de Medicina Interna en los meses de Febrero a Mayo del año 2003, el único criterio de inclusión fue tener una edad $>64$ años.

Se consideró fármaco potencialmente inadecuado para el paciente aquel principio activo que no debe ser administrado al anciano o siendo adecuado se ha prescrito a dosis excesiva o durante un tiempo superior al indicado para ese grupo de pacientes. Para establecer la lista de medicamentos se siguen los criterios de Hanlon JT (Tabla I) (7) y los criterios de Beers y cols., (8) sobre fármacos inadecuados en base a posibles patologías que aparecen en el anciano (Tabla II). Se consideraron así mismo fármacos no indicados aquellos que el paciente tomaba sin que existiese patología alguna que lo justificase, y fármacos de utilidad terapéutica baja (UTB), aquellos principios activos cuya eficacia no ha sido demostrada por ensayos clínicos controlados (Tabla III) (9).

Se analizaron las siguientes variables que podían influir en el resultado final; sexo, edad, procedencia (residencia "R" o domicilio "D"), estado cognitivo, $\mathrm{n}^{\circ}$ de patologías activas según criterio GRD, $n^{\circ}$ de fármacos al ingreso, $n^{\circ}$ de prescriptores en los últimos dos años, aparición de efectos secundarios e ingreso por efectos secundarios. El análisis estadístico se realizó mediante el programa estadístico SPSS 11.5, utilizando el procedimiento"stepwise"del modelo de regresión lineal para el análisis multivariante.

\section{RESULTADOS}

Se analizaron un total de 172 pacientes, el consumo medio de medicamentos fue de 5,34 medicamentos por persona-día (excluyendo los que estuviesen tomando por el cuadro agudo que motivó el ingreso) con una horquilla 0-15. El 52,3\% de los ancianos estudiados consumía al menos un fármaco injustificado (UTB, inadecuado o no indicado).

Dentro de este grupo de fármacos destaca el consumo de fármacos inadecuados para el anciano, $36,6 \%$ con un rango de 0-3, siendo el uso inadecuado de benzodiacepinas el grupo más amplio (23,8 \% de los ancianos). El resto de medicamentos aparece descrito en la figura 1.

El segundo grupo lo constituyen los fármacos no indicados, $15,1 \%$ de la población estudiada, destacando el mal uso de los inhibidores de la bomba de protones, $11 \%$, el uso del alopurinol para la hiperuricemia leve asintomática asociada al uso de diuréticos, o el sulpiride utilizado de forma mantenida para el mareo inespecífico.

Por último describimos el consumo de fármacos UTB, $12,1 \%$ admitía tomar al menos un fármaco de dudosa utilidad terapéutica, siendo la pentoxifilina, citicolina y n-acetilcisteina los más utilizados (Tabla III).

$\mathrm{El}$ análisis univariante del conjunto de estos fármacos establece una asociación del consumo de fármacos injustificados con consumo de mayor cantidad de medicamentos $(\mathrm{p}<0,012)$ y con la procedencia de residencia de ancianos $\mathrm{p}<0,049(65,2 \%$ en los que procedían de residencia frente al $48 \%$ del domicilio),
TABLA I

MEDICAMENTOS INAPROPIADOS EN EL ANCIANO

Antihistamínicos

Clorfeniramina

Maleato de dexclorferinamina

Difenidramina

Prometazina

Inhibidores de la agregación plaquetaria (El riesgo de efectos secundarios es alto)

Ticlopidina

Dipiridamol

Cardiovasculares

Antihipertensivos

Alfametildopa

Reserpina

Propanolol (poco B-selectivo y muchos efectos sobre el SNC)

Hidroclorotiacida a dosis superiores a $50 \mathrm{mg} / \mathrm{dí}$.

Vasodilatadores periféricos

Mesilato de ergotamina

Pentoxifilina.

Antiarrítmicos

Digoxina usada a dosis $>0,125 \mathrm{mg}$ /día salvo en la fibrilación auricular

Fármacos que actúan sobre el SNC

Benzodiacepinas de vida media (vm) corta y larga

Vm larga (diazepam, fluracepam etc. no utilizar nunca)

Vm corta (oxazepam, triazolam, alprazolam, usar sólo en casos necesarios y por

un tiempo inferior a 4 semanas)

Antidepresivos

Amitriptilina

Imipramina

Doxepina por sus efectos anticolinérgicos

Antipsicotivos

Haloperidol

Tioridazina

Combinación de antidepresivos y antipsicotivos

Barbitúricos

Pentobarbital

Secobarbital, (excepto fenobarbital)

Narcóticos

Meperidina

Pentazocina

Propoxifeno

Gastroenterológicos

AntiH2 (ranitidina a dosis > $300 \mathrm{mg}$ y tratamientos superiores a 12 semanas)

Antiespasmódicos (su uso durante tiempo prolongado debe evitarse)

Endocrinológicos

Hipoglucemiantes orales

Clorpropamida

Riesgo alto de SIADH

Musculoesqueléticos

AINES (indometacina, por sus reacciones toxicas sobre el SNC, ketorolaco, piroxicam, fenilbutazona, acido mefenámico)

Tomada de Hanlon JT et al. Ann Pharmacother 2000; 34:362.

no existiendo asociación con el resto de las variables. El análisis multivariante establece una asociación independiente de estos fármacos injustificados con; mayor consumo de medicamentos $(\mathrm{p}<0,001)$, procedencia de residencia de ancianos $(\mathrm{p}<0,001), y$ con el número de diagnósticos al ingreso $(\mathrm{p}<0,012)$.

$\mathrm{Si}$ analizamos mediante análisis multivariante los factores asociados al consumo de cada grupo de estos fármacos los 
TABLA II

EFECTOS ADVERSOS DESCRITOS EN ANCIANOS EN RELACIÓN CON SU PATOLOGÍA DE BASE

\begin{tabular}{|c|c|c|c|}
\hline $\begin{array}{l}\text { AINES } \\
\text { Alfa-bloqueantes } \\
\text { Antagonista Ca++ } \\
\text { Antihistamínicos } \\
\text { Antiespasmódicos } \\
\text { Antidepresivos Tricíclicos } \\
\text { Aspirina } \\
\text { Benzodiacepinas } \\
\text { B-bloqueantes }\end{array}$ & $\begin{array}{c}\text { Insuficiencia cardiaca Insuficiencia } \\
\text { renal, HTA, Ulcera péptica } \\
\text { Incontinencia urinaria } \\
\text { Insuficiencia cardiaca } \\
\text { HBP, Estreñimiento } \\
\text { HBP, Estreñimiento } \\
\text { HBP, Estreñimiento y Claucoma } \\
\text { Ulcera péptica } \\
\text { Demencia, Síncopes } \\
\text { Asma, insuficiencia cardiaca, diabetes, } \\
\text { Raynaud, EPOC, Enfermedad vascular }\end{array}$ & $\begin{array}{l}\text { Clorpromacina } \\
\text { Clozapina } \\
\text { Corticoide sistémico } \\
\text { Descongestionantes } \\
\text { Diurético tiazídico } \\
\text { IMAOS } \\
\text { Inhibidores recaptación } \\
\text { de serotonina } \\
\text { Metoclopramida } \\
\text { Narcóticos } \\
\text { Relajante muscular } \\
\text { Sedantes-Hipnótico } \\
\text { Suplementos de K } \\
\text { Teofilina }\end{array}$ & $\begin{array}{c}\text { Convulsiones, Hipotensión postural } \\
\text { Convulsiones } \\
\text { Diabetes } \\
\text { Insomnio } \\
\text { Cota } \\
\text { Insomnio } \\
\text { Insomnio } \\
\text { Convulsiones } \\
\text { HBP, Estreñimiento } \\
\text { Insomnio } \\
\text { EPOC } \\
\text { Ulcera péptica } \\
\text { Insomnio }\end{array}$ \\
\hline
\end{tabular}

TABLA III

SUBGRUPOS TERAPÉUTICOS CONSIDERADOS DE BAJA UTILIDAD TERAPÉUTICA

Vasodilatadores periféricos

Antivaricosos tópicos

Protectores capilares

Otras vacunas asociadas

AINES tópicos

Otros preparados para el aparato locomotor

Otros psicoanaléticos, excluidos preparados antiobesidad

Expectorantes, incluidos mucolíticos, sin antiinfecciosos

Otros preparados para el aparato respiratorio

Antiespasmódicos y anticolinérgicos gastrointestinales asocidados con otras sustancias

Alcaloides de la rauwolfia asociados con diuréticos

Diuréticos asociados con otras sustancias

Antihemorroidales locales sin corticoides

Antibióticos asociados con sulfamidas tópicas
Corticoides tópicos asociados con antibióticos y antimicóticos

Andrógenos asociados con hormonas femeninas

Antisépticos y antiinfecciosos urinarios en asociación

Corticoides sistémicos asociados con otros

Antibióticos asociados entre si

AINEs asociados con otros

AINEs asociados con corticoides

Miorrelajantes de acción central asociados con otros

Hipnóticos y sedantes en asociación

Corticoides asociados con antiinfecciosos de administración tópica

Psicolépticos asociados con psicoanalépticos

Antiasmáticos asociados con otros

Antiinfecciosos con expectorantes o mucolíticos

Corticoides asociados con antiinfecciosos de aplicación ótica

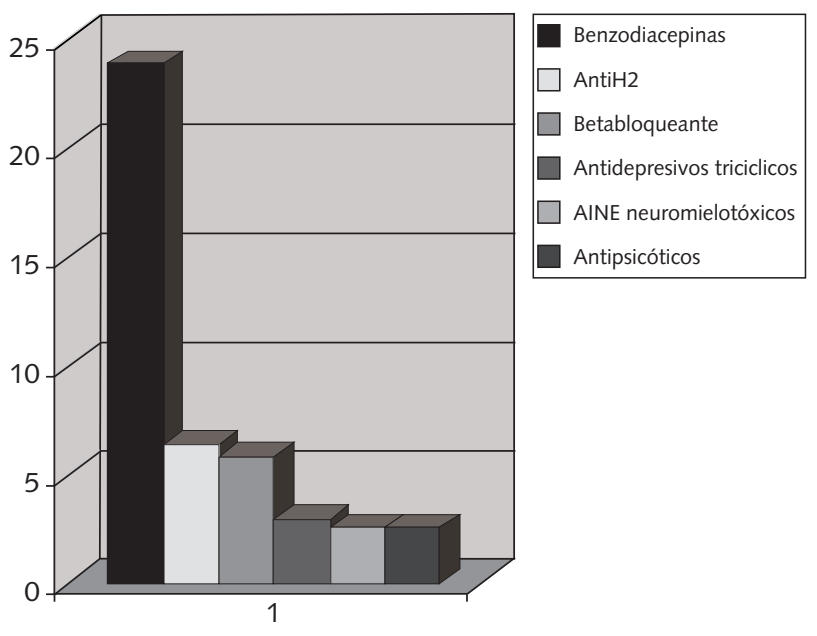

Tipo de fármaco

Fig. 1. Porcentaje de ancianos que consumen distintos tipos de fármacos inadecuados para el anciano. datos obtenidos ofrecen una asociación en el consumo de fármacos inadecuados para el anciano, además de los factores anteriormente descritos, la demencia $(\mathrm{p}<0,004)$ y un mayor ingreso por efectos secundarios $(\mathrm{p}<0,029)$. En el caso de los fármacos no indicados se asocia únicamente al mayor número de ingresos previo $(\mathrm{p}<0,046)$. Por último los fármacos UTB se asocian a la procedencia de residencia de ancianos ( $\mathrm{p}<$ $0,001)$, demencia $(\mathrm{p}<0,001)$ y al consumo de fármacos no indicados o inadecuados $(\mathrm{p}<0,001)$.

\section{DISCUSIÓN}

El consumo de fármacos se ha incrementado en las últimas dos décadas en los países industrializados de forma llamativa, afectando fundamentalmente a la población anciana. En un estudio inglés realizado por Law y cols. (10) en 1976, el 34\% de los ancianos consumían un máximo de 3-4 fármacos, sin embargo en los estudios más recientes, el primero de ellos del año 1997, estiman que la media diaria es 4,2-8 (1-3) fármacos por persona, con un consumo máximo de hasta 18 fármacos distintos al día (3), sin tener en cuenta además el autoconsu- 
mo, muchas veces no reconocido por los pacientes si no son preguntados expresamente al respecto.

Tal vez más llamativo sea lo que representa esto en términos contables. En España el gasto farmacéutico suponía en el año 1991 el 22,24\% del gasto sanitario, en el año 2000 se había incrementado al 31,59\% (11), sin que esto se corresponda con un empeoramiento en los estándares de salud.

Todos los datos apuntan hacia la existencia de un marcado consumo, que dudosamente corresponde a las necesidades de salud de nuestra sociedad. A tenor de lo expuesto surge la siguiente pregunta: ¿es necesario que cada persona llegue a tomar hasta 18 fármacos diferentes al día? Existen pocos estudios a nivel mundial, y en nuestro país en particular, que afronten este tema, destaca el realizado por Fidalgo y cols., (12) que analizaron el consumo de fármacos de una población de ancianos del área 8 de Madrid. Sus datos muestran que el 97\% de los ancianos que vivían en residencias consumían al menos un fármaco de forma injustificada (incluyen aquí fármacos UTB y fármacos inadecuados para el anciano), frente a un $61 \%$ de los ancianos ambulatorios, en las residencias de ancianos en los que se realizaba una intervención sobre los hábitos de prescripción este porcentaje se situaba en el 51\%. Los datos que aporta nuestro estudio establecen la misma conclusión, un marcado consumo de fármacos injustificados en las residencias de ancianos si bien este es del 65,2\% de los pacientes ingresados $v s$. el $48 \%$ de los procedentes de su domicilio.

Existen otros estudios que confirman los datos aquí descritos, como el publicado recientemente por Pittrow D y cols. (13), estudio alemán en el que al analizar las prescripciones realizadas por médicos de atención primaria y por médicos en residencias de ancianos. La media era de 31 por persona-año en el primer grupo, mientras que en las residencias de ancianos era de 37, y esta mayor prescripción era a expensas de medicamentos inapropiados, principalmente psicotropos, analgésicos, laxantes y diuréticos.

Un segundo factor a considerar, que hemos apreciado en nuestro trabajo, y que no hemos encontrado descrito en otros, es el consumo de mayor número de fármacos. Los ancianos que más medicamentos toman por sus patologías son a su vez los que mayor número de fármacos injustificados consumen, fundamentalmente a expensas de los fármacos inadecuados para el anciano y dentro de este grupo los psicotropos, y son también aquellos que más patologías de base tienen los que mas medicamentos injustificados ingieren a diario. Tal vez, y teniendo en cuenta que son las benzodiacepinas los fármacos mas consumidos dentro de esta población, los factores asociados a este consumo sean los expuestos por Gómez MJ y cols., (14) en su análisis sobre la prescripción de benzodiacepinas en atención primaria, si bien se podría aplicar a otros muchos fármacos. Del citado estudio destacaré dos puntos; el principal factor para explicar esta prescripción es la medicalización de la sociedad y sus disminución de la tolerancia al mal estar emocional, el sentirse mal obliga a consumir fármacos ante cualquier síntoma, y si importante es este punto también lo es el segundo, la falta de tiempo en las consultas, "es más fácil prescribir que escuchar".

Un último factor independiente asociado al consumo de fármacos injustificados es la demencia, principalmente factor independiente relacionado con la ingesta de fármacos inadecuados y fármacos UTB. Algunos estudios estiman que el consumo de psicotropos en los ancianos dementes alcanza el $41 \%$ (15), sin embargo es probable que esta cifra esté más próxima al $70 \%$ que ofrece nuestro estudio.
Al analizar de forma independiente cada grupo de fármacos, que constituyen esta familia de fármacos injustificados en el anciano, surgen las tres preguntas básicas que hay que responder al analizar los medicamentos que consumen nuestros pacientes:

-El fármaco que toma ¿tiene alguna utilidad? ¿Existen estudios randomizados que acrediten que esa sustancia química tiene algún efecto beneficioso sobre la salud?.

En la Tabla 3 se recoge el listado de los fármacos denominados de baja utilidad terapéutica (UTB) que agrupan este tipo de sustancias.

Los estudios publicados coinciden con los aquí expuestos. Entre el $12,7 \%$ (16) y el 18,2\% (17) de los ancianos consumen alguno de estos fármacos, siendo los vasodilatadores periféricos los mas ingeridos. No parece existir una incidencia de los mismos sobre la salud, el análisis multivariante no ha demostrado un incremento de los efectos secundarios, ni de ingresos asociados a su ingesta, asociándose está además de a los factores anteriormente expuestos al consumo de fármacos inadecuados para el anciano.

- ¿Necesita el paciente tomar ese fármaco? La respuesta a esta pregunta se basa en un hecho universalmente reconocido. Los medicamentos son diseñados y utilizados con un único propósito, aliviar, prevenir o curar determinadas patologías o síntomas, si no existe este precepto nada justifica su uso. Esto que puede parecer algo descabellado no resulta infrecuente. En un estudio ambulatorio realizado por McDonnell y cols., (18) sobre morbi-mortalidad relacionada con el consumo de medicación, y encaminado a valorar los efectos derivados de la retirada de todos los fármacos posibles, se observó que el motivo esgrimido en el 8,5\% de los casos para la interrupción de la medicación fue el de no tener ninguna indicación en el paciente.

Existen varios motivos por los que un paciente recibe un fármaco no indicado:

- Por un lado el utilizar los fármacos como placebo en pacientes con síntomas vagos o inespecíficos.

-Otorgarle a un fármaco indicaciones que no posee, como utilizar ansiolíticos en el tratamiento mantenido de la hipertensión arterial.

- Mantener de forma indefinida un medicamento una vez finalizado el tiempo estimado para un tratamiento correcto. No es infrecuente que los especialistas que han prescrito el medicamento no vuelvan a evaluar al paciente y el médico de atención primaria no retire el tratamiento. En el citado estudio de McDonell y cols., (18) se estimó que en el 67\% de los efectos adversos estudiados había existido un seguimiento inadecuado de la terapia del paciente por parte del médico.

-Efecto cascada. Bajo este epígrafe se recoge un hecho observado con frecuencia en la medicina y por el cual un determinado factor, generalmente la ansiedad del paciente o del propio médico, inicia un proceso de acontecimientos concatenados con consecuencias previsibles e irreversibles en el paciente, generalmente un daño físico o psíquico (19). En la cascada terapéutica, un fármaco produce un efecto secundario que es tratado con otro fármaco. El ejemplo más habitual son los mareos inespecíficos en muchas ocasiones asociados a la medicación hipotensora que son tratados con sulpiride, que a su vez puede originar un parkinsonismo y recibir el paciente un tercer fármaco como puede ser la l-dopa.

En nuestro estudio el $15,1 \%$ de los ancianos recibe algún fármaco sin que exista una indicación para su utilización en el 
momento del ingreso. El mayor consumo de estos fármacos se da en pacientes procedentes de residencias de ancianos, y con el mayor número de diagnósticos al ingreso.

El fármaco principal incluido en este grupo es el inhibidor de la bomba de protones, el $34 \%$ de lo que tomaban este fármaco, (el $11 \%$ de la población) no lo tomaba bajo ninguna indicación admitida para su uso, en ocasiones mantenidos durante años tras haber sido utilizado como tratamiento para una úlcera, otras para prevenir sangrados al consumir anticoagulantes, corticoides o analgésicos del tipo de los opiáceos. Otros ejemplos de fármacos descritos en este grupo son; el uso persistente de sulpiride para el tratamiento de mareos inespecíficos, o el consumo de cinitaprida administrado crónicamente en el tratamiento de dispepsia. Un caso también frecuente es el tratamiento con alopurinol de la hiperuricemia leve asintomática asociada al uso de diuréticos.

- ¿Es adecuado el consumo de ese fármaco en las condiciones físicas y mentales que tiene el paciente? Para responder a esta última pregunta recurrimos al documento de consenso sobre fármacos inadecuados para el anciano expuesto por Halon y cols., (7) y Beers y cols., (8), cuyas bases son explicadas en el apartado de material y métodos.

Básicamente un fármaco inadecuado para el paciente es aquel que es potencialmente lesivo, pudiendo además evitarse su uso al existir otra alternativa menos perjudicial e igual de efectiva. Puede ser inadecuado por la edad, al aumentar su toxicidad en edades extremas de la vida, (un ejemplo podría venir dado por la condición mental, la cual desaconseja el uso de la mayor parte de fármacos psicoactivos por el aumento de la sedación), o puede serlo por las patologías de base que presente el paciente (ejemplo, usar betabloqueantes en pacientes asmáticos).

Este es el grupo de fármacos más importante entre aquellos que no deben ser tomados por los pacientes estudiados, ya que el $36,6 \%$ de los ancianos tomaban uno o varios de estos fármacos. De acuerdo a los criterios previamente establecidos las benzodiacepinas son los más utilizados, 23,8\% de los ancianos, seguidos por la ranitidina por un $6,4 \%$ de los pacientes ingresados.

El excesivo uso de medicación inapropiada en los ancianos ha sido descrito en otros trabajos $(13,20,21)$ siendo los principales fármacos implicados los Psicotropos (neurolépticos, antidepresivos e hipnóticos), siendo otros fármacos los Analgésicos o la digoxina. Estos datos coinciden con otros estudios. De la importancia de los inadecuado de su prescrip- ción son los datos aportados por Gurwitz (22) al evaluar los efectos secundarios en residencias de ancianos. La mayoría se debieron a las drogas psicoactivas y principalmente a las benzodiacepinas.

Una última faceta que se ha analizado es la influencia de estos medicamentos en la aparición de efectos adversos. Casi todos los estudios sobre polimedicación concluyen que el mayor número de medicamentos se asocia a un incremento en el riesgo de aparición de efectos secundarios (23-26). El hecho de tomar medicamentos de forma innecesaria incrementa pues este riesgo, si bien no de forma directa si por el riesgo de incrementar las interacciones. Evaluados en su conjunto los fármacos injustificados no se asociaban a una mayor incidencia de efectos secundarios, pero si analizamos únicamente los inadecuados para el anciano, excluyendo fundamentalmente los fármacos UTB que al igual que apenas tienen efectos sobre la salud tampoco parecen tenerlo sobre la enfermedad, se evidencia que esa relación existe, observándose en el análisis multivariante la existencia de una asociación independiente del consumo de estos fármacos con un incremento del número de ingresos por efectos secundarios.

La explicación a este hecho podría encontrarse en el consumo de AINES de forma inadecuada y la aparición de HDA, (2,3\% de los ingresos observados), o bien en el consumo de benzodiacepinas que interaccionan al igual que los antiH2 en el citocromo P450 con otros fármacos potenciando sus interacciones, o el consumo de Betabloqueantes en pacientes con asma o bronquitis desencadenando cuadros de reagudización, (1,2\% de los ingresos).

Podemos concluir que el 52,3\% de los ancianos que ingresaron en el Servicio de Medicina Interna consumían un fármaco de forma injustificada, siendo los fármacos inadecuados para el anciano, con un $36,6 \%$ de consumo, el grupo de medicamentos más consumidos, y las benzodiacepinas el tipo de fármacos más importante $23,8 \%$ de los ancianos.

La mayor prescripción de estos fármacos se da en ancianos procedentes de residencia de ancianos, y entre aquellos que más patologías tienen y más fármacos consumen, lo que podría estar en relación con una mala tolerancia física y psíquica al "estrés". La valoración global no muestra un incremento de los efectos adversos, sin embargo el consumo de fármacos inadecuados para el anciano se asocia a un incremento en los ingresos por efectos adversos.

\section{Bibliografía}

1. Graves T, Hanlon JT, Schmader KE, Landsman P, et al. Adverse events after discontinuing medications in elderly outpatients. Arch Intern Med 1997; 157: 2205-10

2. Blasco F, Martínez López de Letona J, Pérez Maestu R, Villares P, Ponce J. Estudio piloto sobre el consumo de fármacos en ancianos que ingresan en un hospital. An Med Intern (Madrid) 2004; 21: 69-71.

3. Hohl CM, Dankoff J, Colacone A, Afilalo M. Polypharmacy, adverse drugs and potential adverse drugs interactions in elderly. Ann Emerg Med 2001; 38: 666-71

4. Fidalgo ML, Molina T, Millan F, Orozco P, Benavente I, Casado M, López C. Prescripción farmacéutica en residencias de ancianos. Comparación con ancianos ambulatorios ( $2^{\mathrm{a}}$ parte). MEDIFAM 2001; 11: 73 82 .
5. Alcalde $\mathrm{P}$, Dapena MD, Nieto MD, Fontecha BJ. Ingreso hospitalario atribuible a efectos adversos a medicamentosos. Rev Esp Geriatr Gerontol 2001; 36: 340-4.

6. Blasco F. Efectos secundarios de la medicación. En Sobremedicación de los pacientes ancianos. Efectos sobre su salud y sobre el sistema sanitario. Tesis doctoral. Universidad Autónoma de Madrid 2004. p. 4950

7. Hanlon JT, Shimp LA, Semla Tp. Recent Advances in Geriatrics: DrugRelated Problems in the Elderly. Ann Pharmacother 2000; 34: 360-365.

8. Beers $\mathrm{MH}$, Explicit criteria for determining potentially innappropiate medications use by the elderly: An update. Arch Intern Med 1997; 157 : 1531-6.

9. Agudo C, Azparren A, Erviti J et al. Medicamentos de utilidad terapéu- 
tica baja. Alternativas a su utilización. Gobierno Navarro. Departamento de Salud. Servicio de Prestaciones Farmacéuticas. Dirección de Atención Primaria. Argibideak. 1996; 9:2-4.

10. Law R, Chalmers C. Medicines and elderly people: A general practice survey. B M J 1976; 1: 565-8.

11. Costas E. Diez años de gasto farmacéutico galopante. SEMERGEN 2001; 27: 348-9.

12. Fidalgo ML, Molina T, Millan F, Orozco P, Benavente I, Casado M, López C. Prescripción farmacéutica en residencias de ancianos. Comparación con ancianos ambulatorios ( $2^{\mathrm{a}}$ parte). MEDIFAM 2001; 11: 73-82.

13. Pittrow D, Krappweis J, Rentsch A, Schindler C, Hach I, Bramlage P, Kirch W, Pattern of prescriptions issued by nursing home-based physicians versus office-based physicians for frail elderly patients in German nursing homes. Pharmacoepidem Drug Safety 2003; 12: 595-9.

14. Gómez MJ, Baides C, Martínez J, Fernández T. Factores que incluyen en la prescripción de benzociacepinas y acciones para mejorar su uso: un estudio Delphi en atención primaria. Aten Primaria 2002; 30: 297 303.

15. Giron MS, Forsell Y, Bernsten C, Thorslund M, Winblad B, Fastbom J Psychotropic drug use in elderly people with and without dementia. Internat J Ger Psyth 2001; 16: 900-6.

16. Besco E, Pérez M, Torrent M, Llach A, Seguí M, Bartolozzi E, Ramos J. Prescripciones de utilidad terapéutica baja inducidas en atención primaria. Aten Primaria 1998; 22: 227-32.

17. Martínez JA, Baeza JE, Rubio-Manzanares JA, Palacios MC. Impacto de un programa de uso racional del medicamento sobre la prescripción farmacéutica en un área de salud. Aten Primaria 1996; 18: 551-7.

18. McDonell JP, Jacobs MR. Hospital admission resulting from preventable adverse drugs reaction. Ann Pharmacother 2002; 36: 1331-13.

19. Mold JV, Stein HF. The cascade effect in the clinical care of patients. N Eng J Med 1986; 314: 512-4.

20. Sørensen L, Foldspang A, Gulmann C, Munk-Jørgensen P. Determinants for the use of psychotropics among nursing home residents. Intern J. Geriat Psychiatry 2001; 16: 147-54.

21. Mayer-Oakes SA, Kelman G, Beers MH, De Jong F, Matthias R, Atchinson A, et al Benzodiazepine use in older, community-dwelling southern californians: prevalence and clinical correlates. Ann Pharmacoth 1993; 27: 416-21.

22. Gurwitz J H, Field TS, Avorn J, McCormick D, Jain S, Eckler M, et al. Incidence and preventability of adverse drug events in nursing homes. Am J Med 2000; 109: 87-94.

23. Gray SL, Mahoney JE, Blough DK. Adverse drug events in elderly patients receiving home health services following hospital discharge. Ann Pharmacother 1999; 33: 1147-53.

24. Beltrami GC, Menegolli GP, Corra L, Weber U, Longobardi A, Albiero A, et al. Drug adverse effects in the elderly hospitalized with acute pathologies Clin Ter 2000; 151: 19-23.

25. Chan M, Nicklason F, Vial JH. Adverse drugs events as a cause of hospital admission in the elderly. Intern Med J 2001; 31: 199-205.

26. Hafner JW, Belknap SM, Squillante MD, Bucheit KA. Adverse drugs events in emergency department patients. Ann Emerg Med 2002; 39: 258-67. 